\title{
A Novel Fragment Recommendation Workflow using Direct and Indirect Transfer of SAR According to Integrated Similarities of Scaffold Motifs and SAR Trends: Application to Identifying Factor Xa Inhibitors
}

\author{
Tsukasa Ishihara*, Kenichi Mori, Ryosuke Munakata, Ayako Moritomo \\ Medicinal Chemistry Research Labs., Drug Discovery Research, Astellas Pharma Inc. \\ 21, Miyukigaoka, Tsukuba, Ibaraki 305-8585, Japan \\ *E-mail: tsukasa.ishihara@astellas.com
}

(Received February 2; accepted February 17; published online March 4, 2017)

\begin{abstract}
Here we report a new drug design workflow that facilitates the transfer of structure-activity relationships (SARs) and recommends alternative fragments from SAR databases. We first prepare two collections of matched molecular series (MMS) comprising a query set of compounds with their SARs and a set derived from reference SAR databases. The second step detects MMS from the reference SAR sources, which identifies profiles similar to a query MMS according to integrated similarities of scaffold shapes and SAR trends. The third step enumerates new compounds with improved activity profiles compared with a query compound computed using a collaborative filtering algorithm. Our workflow detected direct and latent relationships between a query MMS and those derived from the reference SAR sources. Retrospective application of this workflow to the identification of factor Xa inhibitors yielded recommendations with higher predictive accuracy than a conventional quantitative SAR technique. Moreover, potent S1 binding elements were identified using SAR knowledge independent of information about ligand-protein complexes.
\end{abstract}

Key Words: in silico drug design, de novo drug design, data mining, matched molecular pair

Area of Interest: In silico drug discovery 


\section{Introduction}

For decades, drug discovery has relied on the optimization of molecular structures through iterative cycles of analysis, design, synthesis, and biological evaluation. The analysis of structure-activity relationships (SARs) is the standard method used in medicinal chemistry to characterize the relationships between structures and their biological activities. During drug discovery, medicinal chemists often use at least one scaffold comprising their own motifs to apply SAR information to improve compound profiles, hop into external chemical space, or both. Therefore, the transfer of SAR information is an attractive topic for medicinal chemistry.

A typical example of the transfer of SAR information from one chemical motif to others is a worldwide study of factor $\mathrm{Xa}$ (fXa) inhibitors. DX-9065a, which is the progenitor of small-molecule fXa inhibitors, was found to possess an amidine moiety that binds the S1 pocket [1], and numerous structurally similar amidine derivatives were investigated as well [2]. Other studies of fXa inhibitors revealed that anthranilamide derivatives with a 4-methoxyphenyl group occupy the S1 binding pocket of fXa [3-5]. These studies led numerous researchers to study the replacement of amidine moieties in the fXa inhibitor motifs with non-amidines such as 4-methoxyphenyl, 4-chlorophenyl, or 5-chloropyridyl groups [2].

The rapid development of SAR databases (e.g. ChEMBL) has accelerated the accumulation of data [6]. Computational methodologies to utilize SAR information are progressing toward the incorporation of a fundamental procedure called matched molecular pair (MMP) analysis [7]. MMP analysis detects pairs of molecules that differ in structure at a specific site and reveal relationships between chemical modifications and changes in their biological profiles. Computational MMP analysis is analogous to the standard SAR analysis and accordingly assists medicinal chemists to interpret search results. MMP analysis is therefore a prominent topic in medicinal chemistry as well as in chemoinformatics. Pharmaceutical companies have applied the paradigm to mining of their SAR databases and share their expertise of drug design process [8-10]. As one example, Astellas has developed an MMP-based automated knowledge mining and sharing system named Fragment MAP [11].

MMP analysis has expanded through the use of matched molecular series (MMS), which employs a pairwise concept to analyze a series of related molecules [12]. The Matsy algorithm searches a series of fragments present in SAR databases according to the rank order of activity profiles and proposes alternative fragments [13]. Bajorath reported that matrices of SAR information were used to estimate profiles of newly designed compounds through summing the activity profiles of related neighborhood compounds in the matrices [14]. However, the Matsy procedure only detects a fragment series that is directly related to the series of query fragments, and Bajorath's method focuses on their scaffolds of MMP relationships.

Here we report a new fragment recommendation workflow that is executed in the following steps: (1) preparation of two MMS collections from a query compound set defined using a SAR study and reference SAR databases; (2) detection of MMS from reference sources, which have profiles similar to a query MMS according to integrated indices of two-dimensional (2D) scaffolds and SAR profiles; and (3) estimation of activity profiles using a collaborative filtering algorithm [15]. Our computational procedure directly mines SAR knowledge and also identifies indirect relationships between a query MMS and a reference MMS. Retrospective application of our workflow to investigate S1 binding elements of fXa inhibitors identified alternative fragments, which are reported to occupy the target pocket according to SAR knowledge independent of information about ligand-protein complexes. 


\section{Materials and Methods}

\subsection{Datasets}

We extracted 87 articles describing 1,855 human fXa inhibitors from ChEMBL version 20 according to the following three conditions: (1) inhibitors with inhibitory activities of $\mathrm{IC}_{50}$ values were selected; (2) sugar derivatives and tri- or polypeptides were excluded to focus synthetic inhibitors; and (3) papers included at least three compounds to compute correlation of rank order among MMS. $\mathrm{IC}_{50}$ values were logarithmically converted to $\mathrm{pIC}_{50}$, and the compound set was divided into a query-compound set of 25 derivatives of darexaban (ChEMBL DOC ID; 60,277) [16] and a set of other fXa inhibitors as references.

\subsection{MMS Identification}

MMS is an extension of MMP (Figure 1). MMP analyses were performed for every article, because each article includes independently determined assay conditions, and unification without normalization to standard compounds causes statistical errors. The pairs were aggregated as index scaffolds such that one MMS comprised a series of compounds that included a common scaffold and differed by structural replacements at a single site. To compute the correlation of rank order among MMS in the following step, only MMS with at least three fragments were collected. MMP fragmentation was calculated using the "Matched Molecular Pairs" component of Pipeline Pilot [17] with maximum fragment size $=15$ and minimum core size $=5$.

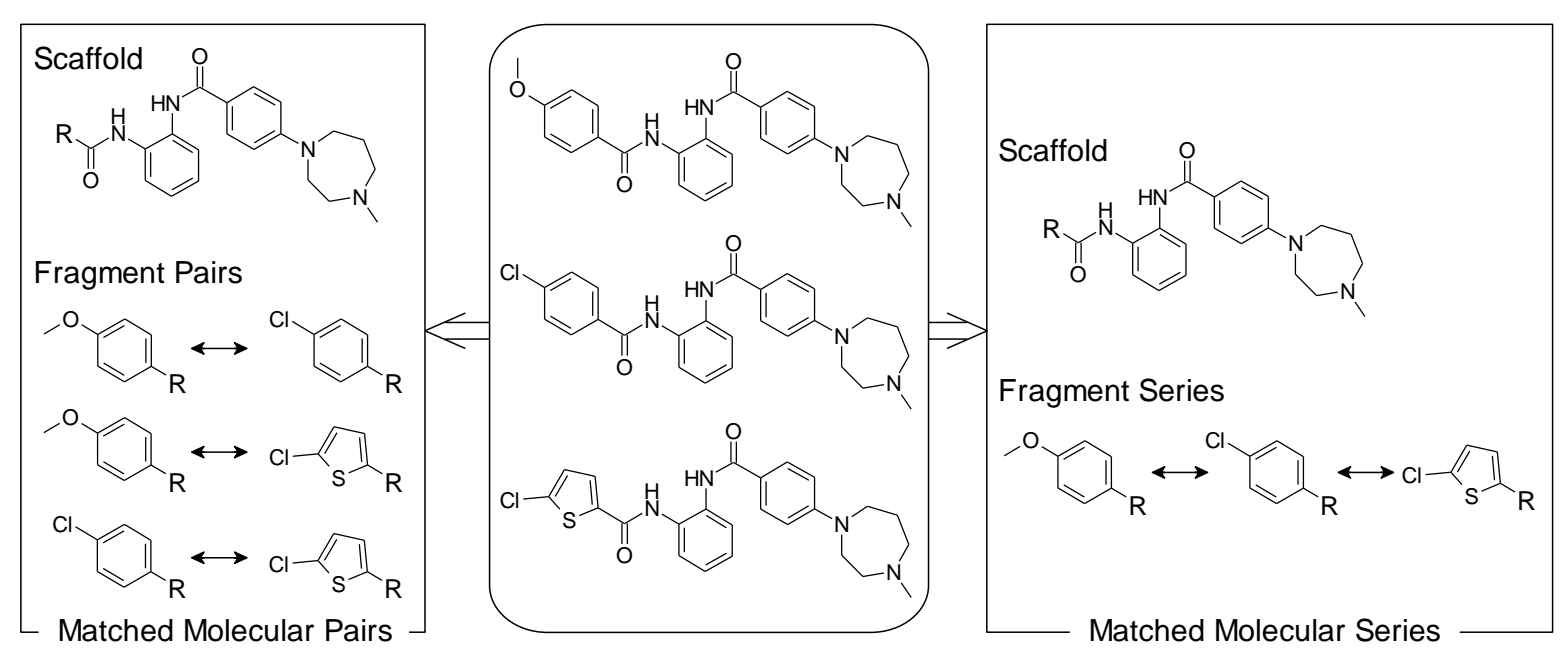

Figure 1. Matched molecular series analysis

\subsection{Detection of Similar MMS using a Direct Search}

The direct search procedure detects MMS with profiles similar to a query MMS according to the immediate relationships among reference SAR sources. To quantitatively evaluate similarities among MMS, we propose a new type of index "MMS similarity". MMS similarity is an integrated concept of two types of similarities. The first one is the similarity of SAR trends according to chemical modifications of fragments, and the other is similarity among scaffolds including the attachment points of fragments. MMS similarity is calculated as the arithmetic mean of the values of the SAR trend and scaffold similarities. 
SAR trend similarity represents the magnitude of an analogy between the activity profiles of two fragment series, which was computed using an activity's rank order as correlation coefficient. $\mathrm{R}$ statistical software was used to compute the correlations [18]. The significance of the difference between the correlation coefficients of two series depends on the number of samples. Therefore, we defined the values of 1- $p$ (1 minus $p$ ) as indices for the SAR trend similarity. MMS with an inverse correlation $(\rho<0)$ were excluded from the subjects for detection because of their dissimilarities among SAR profile trends.

We demonstrate the concept of SAR trend similarity using our previously reported fXa inhibitors (Table 1). Entry A is a collection of YM466 derivatives [19]. Entry B is a set of analogous naphtoanilide derivatives [20], and entry $\mathrm{C}$ contains relative 1,4-diazepan derivatives [21]. In the YM466 series, the SARs of fXa inhibitory activities around the central groups were as follows: 4-carboxyphenyl > carboxymethyl > methyl (entry A). The SAR trend of the naphtoanilide series was equal to that of the YM466 series (entry B). In contrast, the set of 1,4-diazepan derivatives exhibited a different SAR trend: the rank order of activities for the YM466 series was carboxymethyl > methyl > ethoxycarbonylmethyl (entry A), and that for the 1,4-diazepan series was carboxymethyl > ethoxycarbonylmethyl > methyl (entry C). Spearman's rank correlation coefficients $(\rho=1.00$ and 0.50$)$ were calculated for entries B and C vs entry A, respectively, and $p$ values (0.00 and 0.67) were calculated for entries B and C vs entry A, respectively. The SAR trend similarity values (1.00 and 0.33 ) for entries B and C vs entry A were computed, respectively, which enabled quantitative comparison of SAR trends between entries B and C, and revealed that the SAR trend of entry B was more similar to that of entry A than that of entry $\mathrm{C}$.

Table 1. Factor Xa Inhibitors and their SAR Trends

NT: Not Tested. NA: Not Analyzed. -: Not Applicable. Figures in parentheses represent $p$-values.

Entry

The concept of scaffold similarity is based on the traditional "lock and key" model. Two compounds with highly similar structures will arrange their branched fragments in relatively similar spatial positions, and therefore the fragments may function as isosteres. We focused on 2D scaffolds to permit simple calculations. To preserve the orientation of a fragment on a scaffold, it was important to recognize its position on the scaffold. Accordingly, a scaffold was converted to a carbon framework with an attachment point that is replaced with a phosphorus atom to be 
distinguished using molecular modeling software (Table 2). A similarity value between transformed frameworks was computed using the Tanimoto distance with Morgan fingerprints (1,024 bits; radius $=2$ ) using the RDkit extension [22] of the KNIME analytical workflow [23].

Table 2 summarizes scaffold and MMS similarity values. Scaffolds of the YM466 series (entry A) and the naphtoanilide series (entry B) formed a common framework, which was different from that derived from the 1,4-diazepan series in point of their terminal aliphatic rings (entry C). Scaffold similarities $=1.00$ and 0.88 were calculated for the frameworks of entries B and C vs entry A, respectively. The SAR trends and scaffold similarities yielded MMS similarities $=1.00$ and 0.61 for entries B and C vs entry A, respectively.

Table 2. Factor Xa Inhibitors and their Scaffold and MMS Similarities -: Not Applicable.

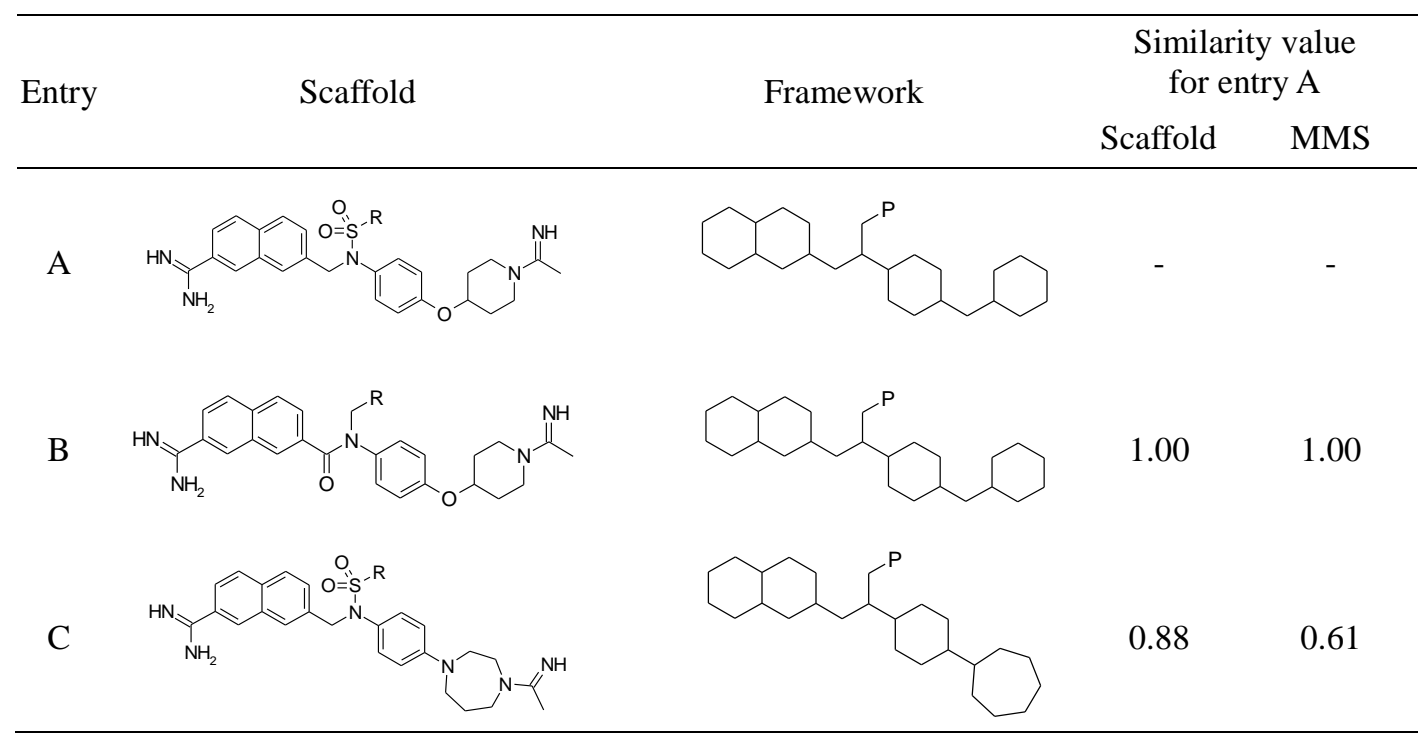

\subsection{Latent MMS Detection using an Indirect Search}

The direct search methodology described in the previous section only detects MMS that include at least three directly related fragments shared by those of a query MMS. In this section, we describe an advanced search protocol that enables researchers to identify related MMS in multi-generation relationships.

An indirect MMS search refers to the analysis of a network structured through the relationships of the MMS. In this network, the nodes represent the MMS, and its edges comprise the relationships of the MMS weighted with MMS similarity values. Analysis of this network detected pairs of MMS through multi-generation relationships, and the calculations of distances between the two related nodes determined the overall similarity values of the pairs of MMS. Searches for related pairs of nodes in a network and distance calculations were performed using $\mathrm{R}$ statistical software with the igraph package [24].

Table 1 describes our concept of indirect MMS relationships. There were only two common elements between the fragments in the naphtoanilide series (entry B) and those in the 1,4-diazepan series (entry C), which prevented researchers from calculating a direct correlation coefficient between the SAR trends of the naphtoanilide and 1,4-diazepan series. In contrast, our search protocol computes an indirect correlation coefficient between entries B and C using entry A as an intermediate. Here, a SAR trend similarity $=0.33$ was computed between entries $\mathrm{B}$ and $\mathrm{C}$ as the mathematical product of a SAR trend similarity value between entries A and B and that between entries $\mathrm{A}$ and $\mathrm{C}$. 


\subsection{Fragment Recommendation with Analogical Estimation of the SAR Profile}

Fragment recommendation is the detection of related MMS by analyzing activity trends followed by the selection of fragments which expected to show higher activity than that of the query compounds. Direct and indirect MMS detection are applicable. Fragment recommendation was performed using preparation of a matrix which tabled SAR trends followed by imputation of the matrix. Matrix preparation was executed as follows: sets of detected MMS were entered in matrix form with their rows and columns corresponding to scaffolds and fragments, respectively (Table 1). We call this MMS matrix, in which an intersection of a row and a column represents one compound. A measured value of a compound is assigned to the corresponding cell. In numerous cases of drug discovery process, MMS matrices include missing values, because not all possible combinations of scaffolds and fragments are generated.

Estimation of missing values refers to activity predictions of newly enumerated compounds. In medicinal chemistry, activity changes are simulated using linear combination of the effects on chemical modifications of scaffolds and fragments, which is applied as the conventional Free-Wilson QSAR analysis [25, 26]. Estimation of missing values in MMS matrices is therefore computed using the additive reflection of SAR trends in their relative rows and columns. Because of the essential consistency of the additive effects of estimation, this protocol can be implemented using collaborative filtering algorithms in the field of recommender system. Simple protocols are available for collaborative filtering, such as GroupLens [27] and Slope One [28], which are classified as user-user and item-item types, respectively. A user-user type provides advantages compared with an item-item type in capitalizing on serendipity [29], and we therefore adopted GroupLens for our profile estimations. The GroupLens calculation was performed using the original source code [27] on R statistical software.

We demonstrate the concept of fragment recommendation using the examples described in Table 1. In these examples, the fragment set of the 1,4-diazepan series comprises methyl, ethoxycarbonylmethyl, and carboxymethyl groups, although carboxyethyl nor 4-carboxypheny group is not included (entry C). Therefore, carboxyethyl and 4-carboxyphenyl analogues in the 1,4-diazepan series were objects for activity estimation as novel compounds. Our fragment recommendation protocol using a GroupLens calculation for entries A with $\mathrm{C}$ directly yielded an estimated $\mathrm{pIC}_{50}$ value $=9.71$ for a 4-carboxyphenyl derivative of 1,4-diazepan series. A GroupLens calculation estimated $\mathrm{pIC}_{50}$ value $=8.11$ for a carboxyethyl derivative of 1,4-diazepan series in the latent relationships observed among entries A, B, and C. A comparative Free-Wilson QSAR analysis computed estimated $\mathrm{pIC}_{50}$ values $=9.10$ and 8.53 for 4-carboxyphenyl and carboxyethyl derivatives of 1,4-diazepan series, respectively, using SAR information transferred in a Free-Wilson matrix followed by multiple linear regression analysis.

\subsection{Comparison with the Conventional Ensemble Bayesian Binary QSAR Model}

We selected 1,855 fXa inhibitors extracted from ChEMBL version 20 as the reference set. An activity threshold of positive or negative values for classification was defined as $\mathrm{IC}_{50}=54.6 \mathrm{nM}$ for the inhibition of fXa activity by darexaban [16]. The ratio between positive or negative samples was 873:982. RDkit Morgan fingerprints (1,024 bits, radius = 2) were calculated for the molecules, followed by 10 -fold cross-validated fitting using the naive Bayes classifier node [30] of KNIME. To generate an ensemble Bayesian model, we performed 500 iterative cycles of model construction with random sampling of cross-validation steps. The model yielded a mean accuracy $=0.74$.

Compound enumeration was executed using a RECAP-type procedure [31]. The rotatable amido bonds of the $\mathrm{fXa}$ inhibitors in the reference set were degraded to generate 1,925 carboxylic acid fragments. We excluded fragments that were previously introduced as S1 binding elements of 
darexaban analogues, and replaced the 4-methoxybenzoyl moiety of darexaban with the degraded fragments followed by filtering using a molecular weight cutoff $\leq 500$, which yielded 40 virtual compounds. Molecular fingerprints of the enumerated compounds were computed using the same method as the modeling step, and activity classification was executed using the constructed ensemble Bayesian model.

\section{Results and Discussion}

\subsection{MMS Identification}

Table 3 shows six representative MMS (IDs = Q1-Q6) generated from 25 darexaban derivatives, from which we defined MMS Q1 as a query series for S1 binding fragments. We identified 750 MMS (IDs = R1-R750) derived from the reference collection of fXa inhibitors.

Table 3. Representative Set of MMS Derived from Darexaban Derivatives

Q6




\subsection{Detection of Similar MMS using a Direct Search}

Table 4.a summarizes MMS relevant to the query MMS using the SAR trend, scaffold, and MMS similarities (entries 1-4), and Tables 5.a-5.d present the details for chemical structures and their fXa inhibitory activities. We found that MMS R1 [32] was most similar to the query MMS Q1 (entry 1 in Table 4.a). Table 5.a shows the detail of comparison between MMS Q1 and R1. The rank order of fXa inhibitory activities in MMS R1 was identical to that of MMS Q1 (Spearman's correlation coefficient $\rho=1.00$ and SAR trend similarity $=1.00$ ). Two scaffolds in this pair differed in their outlying ring systems as follows: the scaffolds of MMS Q1 and R1 formed bicyclic and fused-ring systems, respectively, and varied in their tether lengths to attachment points. The difference between the two scaffolds was calculated as a scaffold similarity $=0.53$, which yielded an MMS similarity $=0.76$.

Table 4.a. Direct Relationships among MMS in the Collection of fXa Inhibitors

\begin{tabular}{ccccccc}
\hline \multirow{2}{*}{ Entry } & MMS & \multicolumn{2}{c}{ SAR Rank } & \multicolumn{3}{c}{ Similarity Value } \\
& Relationship & \multicolumn{2}{c}{ Correlation } & \multicolumn{3}{c}{ S } \\
& & $\rho$ & $p$ & SAR Trend & Scaffold & MMS \\
\hline 1 & Q1-R1 & 1.00 & 0.00 & 1.00 & 0.53 & 0.76 \\
2 & Q1-R2 & 1.00 & 0.00 & 1.00 & 0.36 & 0.68 \\
3 & Q1-R3 & 0.50 & 0.67 & 0.33 & 0.63 & 0.48 \\
4 & Q1-R4 & 0.50 & 0.67 & 0.33 & 0.52 & 0.42 \\
5 & R3-R5 & 1.00 & 0.00 & 1.00 & 1.00 & 1.00 \\
6 & R4-R6 & 0.80 & 0.20 & 0.80 & 0.59 & 0.70 \\
7 & R3-R7 & 1.00 & 0.00 & 1.00 & 0.35 & 0.67 \\
8 & R2-R8 & 0.80 & 0.20 & 0.80 & 0.53 & 0.67 \\
9 & R3-R9 & 0.87 & 0.33 & 0.67 & 0.45 & 0.56 \\
10 & R2-R10 & 0.50 & 0.67 & 0.33 & 0.38 & 0.35 \\
11 & R2-R11 & 0.20 & 0.80 & 0.20 & 0.37 & 0.28 \\
\hline
\end{tabular}

The MMS similarity value between MMS Q1 and R2 [33] = 0.68 was the second highest (entry 2 in Table 4.a). The MMS pair possessed three common fragments that showed identical orders of activity rank $(\rho=1.00)$. The scaffold of MMS R2 differed significantly from that of MMS Q1 (scaffold similarity $=0.36$ ). MMS R3 [34] was the third most similar compared with MMS Q1 (MMS similarity $=0.48$, entry 3 in Table 4.a). The resemblance between the two scaffolds of MMS Q1 and R3 yielded the highest scaffold similarity using our direct search protocol. The scaffold of MMS Q1 formed two directly connected rings, one linkage of two atoms, one ring, and an attachment point through one linkage of two atoms. In contrast, the scaffold of MMS R3 was framed by a motif of two directly connected rings, one linkage of two atoms, one fused ring, and an attachment point through one linkage of one atom. MMS R4 [35] was related to MMS Q1 with an MMS similarity $=0.42$ (entry 4 in Table 4.a). The activity trend of MMS R4 was similar to that of the third-closest MMS R3 with a SAR trend similarity $=0.33$, although the scaffold similarity between MMS Q1 and R4 was lower than that between MMS Q1 and R3.

Our workflow detected immediate relationships among the reference MMS collection automatically, which are additionally listed in Table 4.a. The MMS R3 and R5 [34] pair was a twin series with a combination of identical 2D-scaffold shapes and parallel SAR trends (MMS similarity $=1.00$, entry 5 in Table 4.a). The MMS R4 and R6 [36] pair yielded an MMS similarity $=0.70$ (entry 6 in Table 4.a). The SAR trend of MMS R3 paralleled that of MMS R7 [37] (entry 7 in Table 
4.a), although two scaffolds in this pair differed significantly from each other (scaffold similarity $=$ 0.35, MMS similarity =0.67). Other relationships between MMS pairs R2-R8 [38] (entry 8 in Table 4.a), R3-R9 [38] (entry 9 in Table 4.a), R2-R10 [34] (entry 10 in Table 4.a), and R2-R11 [39] (entry 11 in Table 4.a) were detected with MMS similarities $=0.67,0.56,0.35$, and 0.28 , respectively.

\subsection{Detection of Latent MMS using an Indirect Search}

The query MMS Q1 exhibited a two-generational relationship to MMS R5 through R3 with an overall MMS similarity $=0.48$, which was computed as the mathematical product of the MMS similarity values for MMS Q1-R3 and R3-R5 (entry 4 in Table 4.b). Likewise, MMS Q1 showed an indirect relationship to MMS R8 through R2 with an overall MMS similarity $=0.45$ (entry 5 in Table 4.b). MMS R3 functioned as a hub in the network and connected MMS Q1 to R7 and R9 with overall similarities $=0.32$ and 0.27 , respectively (entries 7 and 9 in Table 4.b). An indirect relationship between MMS Q1 and R6 through R4 had an overall MMS similarity $=0.30$ (entry 8 in Table 4.b). We mined the two-generational relationships between MMS Q1 and R10, and also Q1 and R11 via R2 with overall MMS similarities $=0.24$ and 0.19 , respectively (entries 10 and 11 in Table 4.b).

Table 4.b. Multi-generation Relationships of MMS in the Collection of fXa Inhibitors

\begin{tabular}{ccc}
\hline Entry & $\begin{array}{c}\text { MMS } \\
\text { relationship }\end{array}$ & $\begin{array}{c}\text { Overall MMS similarity } \\
\text { for query MMS Q1 }\end{array}$ \\
\hline 1 & Q1-R1 & 0.76 \\
2 & Q1-R2 & 0.68 \\
3 & Q1-R3 & 0.48 \\
4 & Q1-R3-R5 & 0.48 \\
5 & Q1-R2-R8 & 0.45 \\
6 & Q1-R4 & 0.42 \\
7 & Q1-R3-R7 & 0.32 \\
8 & Q1-R4-R6 & 0.30 \\
9 & Q1-R3-R9 & 0.27 \\
10 & Q1-R2-R10 & 0.24 \\
11 & Q1-R2-R11 & 0.19 \\
\hline
\end{tabular}

\subsection{Characteristics of Detected MMS}

Our workflow mined MMS collections by searching using information only about scaffold motifs and SAR trends without information about including the structures of ligand-protein complexes. However, the detected MMS with high MMS similarity values are reported as S1 binding elements of fXa in the original papers. A previous X-ray crystallographic analysis around MMS R1 (Table 5.a), which was found as the most similar MMS to the query MMS, shows that 4-chloropheny moiety occupies the $S 1$ pocket of fXa [32]. Our workflow also detected the 5-chloro-2-thiophenyl moiety as the related group to the query MMS (MMS R4, Table 5.d), and another previous X-ray crystallographic analysis shows that this moiety is involved in the S1 pocket of fXa [35]. The chloro-substituted thiophene in MMS R6, which showed a two-generational relationship to the query MMS (Table 5.f), interacts with the S1 cavity of fXa in an X-ray structure of a 2-aminomethylphenylamine derivative [36]. Our system yielded four other related series MMS R3, R5, R7, and R8 (Tables 5.c, 5.g, and 5.h), in which the 5-chloro-2-thiopheny moieties of MMS R3 and R5, the 3-amidinophenyl moiety of MMS R7, and the 2-methylbenzofuran moiety of MMS 
R8 were previously modeled as S1 binding elements though computational docking simulation [34, 37, 38].

These results reveal that series of fragments that are located in a certain binding pocket may demonstrate a close SAR trend even when installed in different scaffolds. Consequently, similarity searches of SAR databases according to scaffold frameworks and SAR trends can detect alternative fragments which occupy a particular pocket and function as isosteres. In the present study, fragments of an MMS with an overall MMS similarity $\geq 0.30$ occupied a target S1 cavity.

\subsection{Activity Estimation}

Tables 5.a-5.d show recommended S1 binding fragments of darexaban with inhibitory activity values estimated using our direct search procedure. A SAR trend of MMS R1 was transferred to the query MMS, which estimated a $\mathrm{pIC}_{50}$ value $=6.82$ of a novel darexaban derivative incorporating 5-chloro-2-pyridyl moiety as an S1 binder using a GroupLens calculation (Table 5.a). Likewise, sets of SAR information about MMS R2 and R4 were introduced into the query MMS, which indicated the 3-methoxy and 5-methyl-2-thienyl moieties as alternatives to the S1 binding elements with $\mathrm{pIC}_{50}$ values $=6.96$ and 6.41, respectively (Tables 5.b and 5.d). Although these fragments were predicted to show less potent activities, knowledge transfer from MMS R3 estimated improved fXa inhibitory activity for a new 4-chlorobenzyl analogue $\left(\mathrm{pIC}_{50}=8.44\right)$ compared with that of darexaban (Table 5.c).

Tables 5.e-5.k list the estimated fXa inhibitory activity values for sets of MMS determined using our latent search protocol followed by GroupLens computations. Latent relationships between MMS Q1 and R7 through R3 estimated derexaban derivatives incorporating 3-aminomethylphenyl and 3-amidonophenyl fragments as potent $\mathrm{fXa}$ inhibitors with $\mathrm{pIC}_{50}$ values $=7.57$ and 9.31, respectively (Table 5.g).

Table 5.a. Representative Fragments with fXa Inhibitory Activities Derived from a Phenylpropionylamide Series

Figures in parentheses represent estimated values. The inhibitory activities range from low (red) to high (green) activity.

\begin{tabular}{|c|c|c|c|c|c|c|}
\hline $\begin{array}{l}\text { MMS } \\
\text { ID }\end{array}$ & Scaffold & Framework & 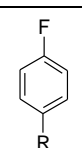 & $\underbrace{C l}_{N}$ & $S_{1}^{c 1}$ & rr \\
\hline Q1 & & & 6.46 & $(6.82)$ & 7.19 & 7.22 \\
\hline R1 & & & 8.00 & 8.64 & 8.89 & 8.92 \\
\hline
\end{tabular}


Table 5.b. Representative Fragments with fXa Inhibitory Activities Derived from an Acylguanidine Series

See the corresponding footnotes of Table 5.a.

\begin{tabular}{|c|c|c|c|c|c|c|c|}
\hline $\begin{array}{c}\text { MMS } \\
\text { ID }\end{array}$ & Scaffold & Framework & & & 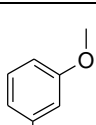 & $\mathrm{A}^{\mathrm{Cl}}$ & \\
\hline Q1 & & & 6.46 & $(6.84)$ & (6.96) & 7.19 & 7.26 \\
\hline $\mathrm{R} 2$ & & & 6.43 & 6.59 & 6.71 & 6.53 & 6.64 \\
\hline
\end{tabular}

Table 5.c. Representative Fragments with fXa Inhibitory Activities Derived from a Tetrahydroisoquinoline Series

See the corresponding footnotes of Table 5.a.

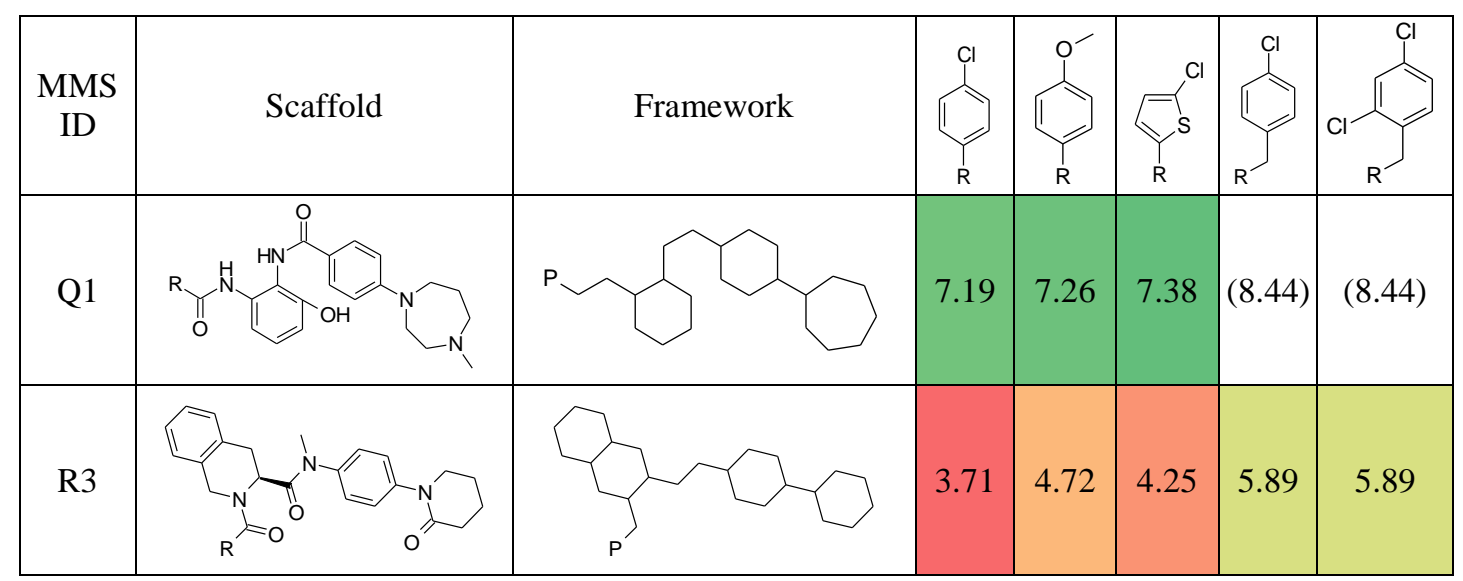

Table 5.d. Representative Fragments with fXa Inhibitory Activities Derived from an Oxazolidinone Series

See the corresponding footnotes of Table 5.a.

\begin{tabular}{|c|c|c|c|c|c|c|c|}
\hline $\begin{array}{c}\text { MMS } \\
\text { ID }\end{array}$ & Scaffold & Framework & ${ }_{Y_{\mathrm{R}}^{\mathrm{H}}}^{\mathrm{H}_{2} \mathrm{~N}}$ & $\underbrace{S}_{R}$ & $\underbrace{c l}_{R}$ & $\bar{Y}_{\mathrm{R}}^{\mathrm{S}}$ & $\Psi_{\mathrm{R}}$ \\
\hline Q1 & & & $(6.10)$ & $(6.41)$ & 7.19 & 7.38 & 7.38 \\
\hline $\mathrm{R} 4$ & & & 8.07 & 8.38 & 7.70 & 9.40 & 9.15 \\
\hline
\end{tabular}


Table 5.e. Representative Fragments with fXa Inhibitory Activities Derived from a Tetrahydroisoquinoline Series

See the corresponding footnotes of Table 5.a. NT: Not Tested.

\begin{tabular}{|c|c|c|c|c|c|c|c|c|}
\hline $\begin{array}{c}\text { MMS } \\
\text { ID }\end{array}$ & Scaffold & Framework & cl & & & & cl & CI \\
\hline Q1 & & & 7.19 & 7.26 & $(7.34)$ & 7.38 & $(8.44)$ & $(8.44)$ \\
\hline R3 & & & 3.71 & 4.72 & 4.79 & 4.25 & 5.89 & 5.89 \\
\hline R5 & & & NT & NT & 4.89 & 4.44 & 5.96 & NT \\
\hline
\end{tabular}

Table 5.f. Representative Fragments with fXa Inhibitory Activities Derived from a Benzylaminocarbonyl Series

See the corresponding footnotes of Table 5.e.

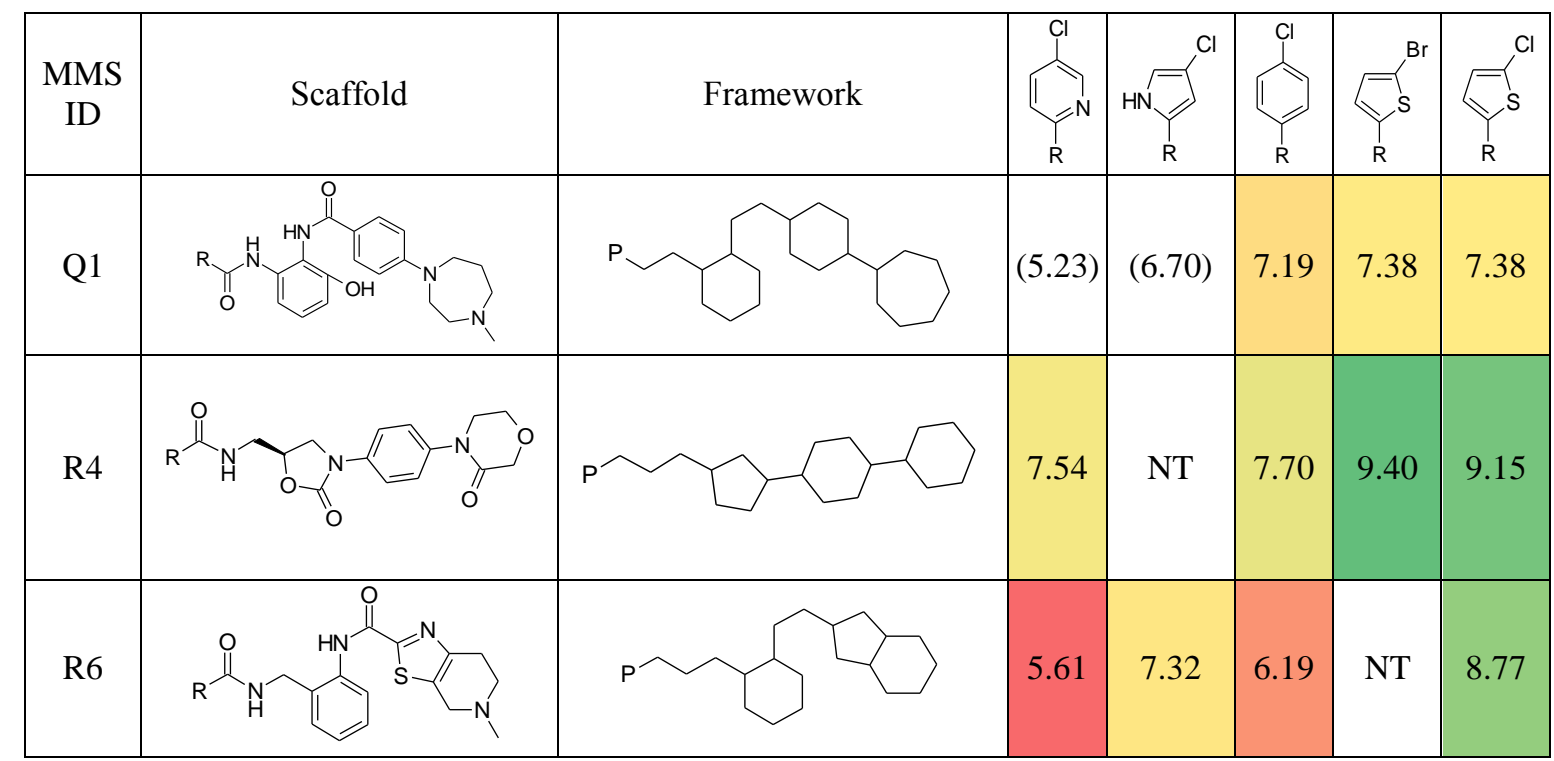


Table 5.g. Representative Fragments with fXa Inhibitory Activities Derived from a Benzoxazinone Series

See the corresponding footnotes of Table 5.e.

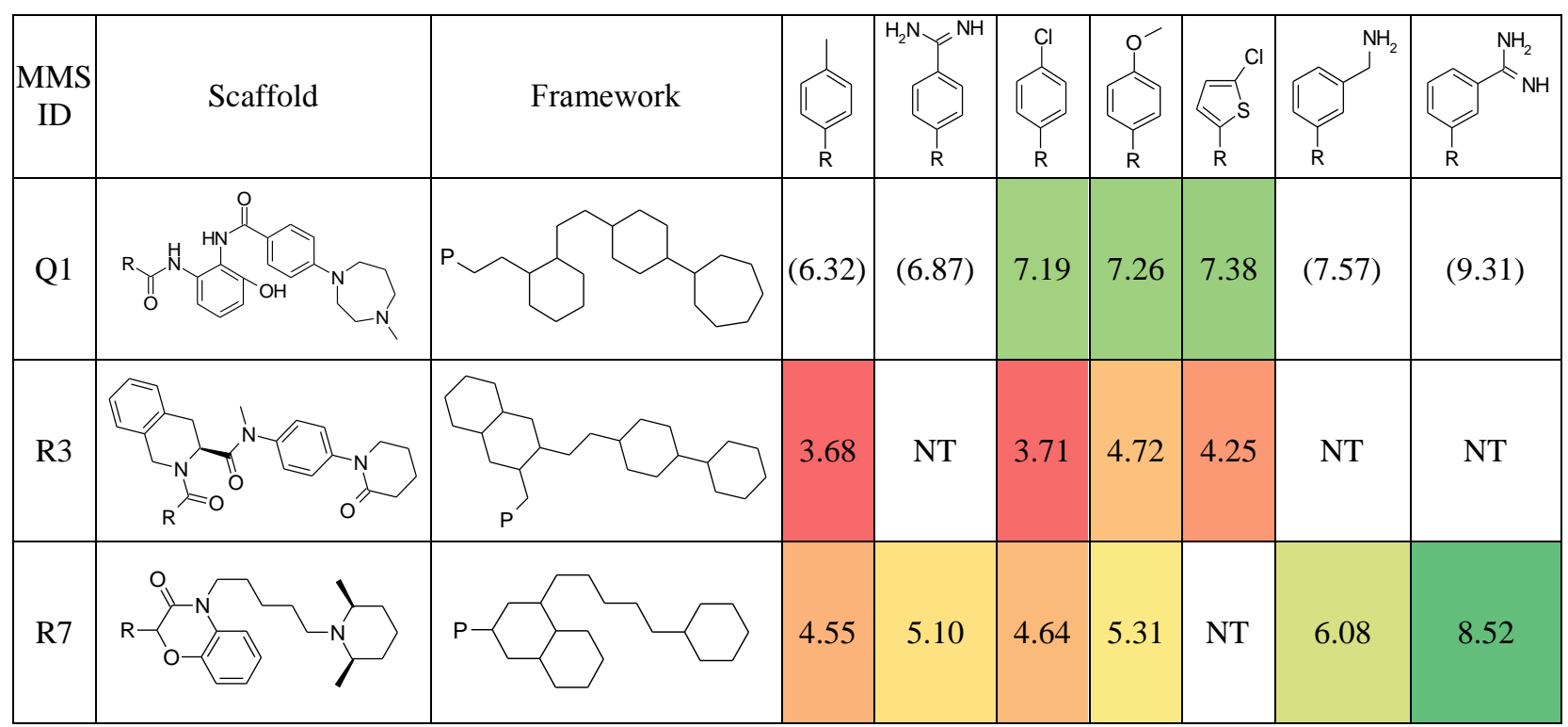

Table 5.h. Representative Fragments with fXa Inhibitory Activities Derived from a Ketene Aminal Series

See the corresponding footnotes of Table 5.e.

\begin{tabular}{|c|c|c|c|c|c|c|c|c|}
\hline $\begin{array}{c}\text { MMS } \\
\text { ID }\end{array}$ & Scaffold & Framework & $\stackrel{F}{d}$ & & & & cl & 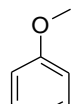 \\
\hline Q1 & & & 6.46 & (6.78) & (6.78) & $(6.96)$ & 7.19 & 7.26 \\
\hline R2 & & & 6.43 & NT & NT & 6.71 & 6.53 & 6.64 \\
\hline R8 & & & NT & 7.29 & 6.27 & 5.17 & NT & 5.51 \\
\hline
\end{tabular}


Table 5.i. Representative Fragments with fXa Inhibitory Activities Derived from a Ketene Aminal Series

See the corresponding footnotes of Table 5.e.

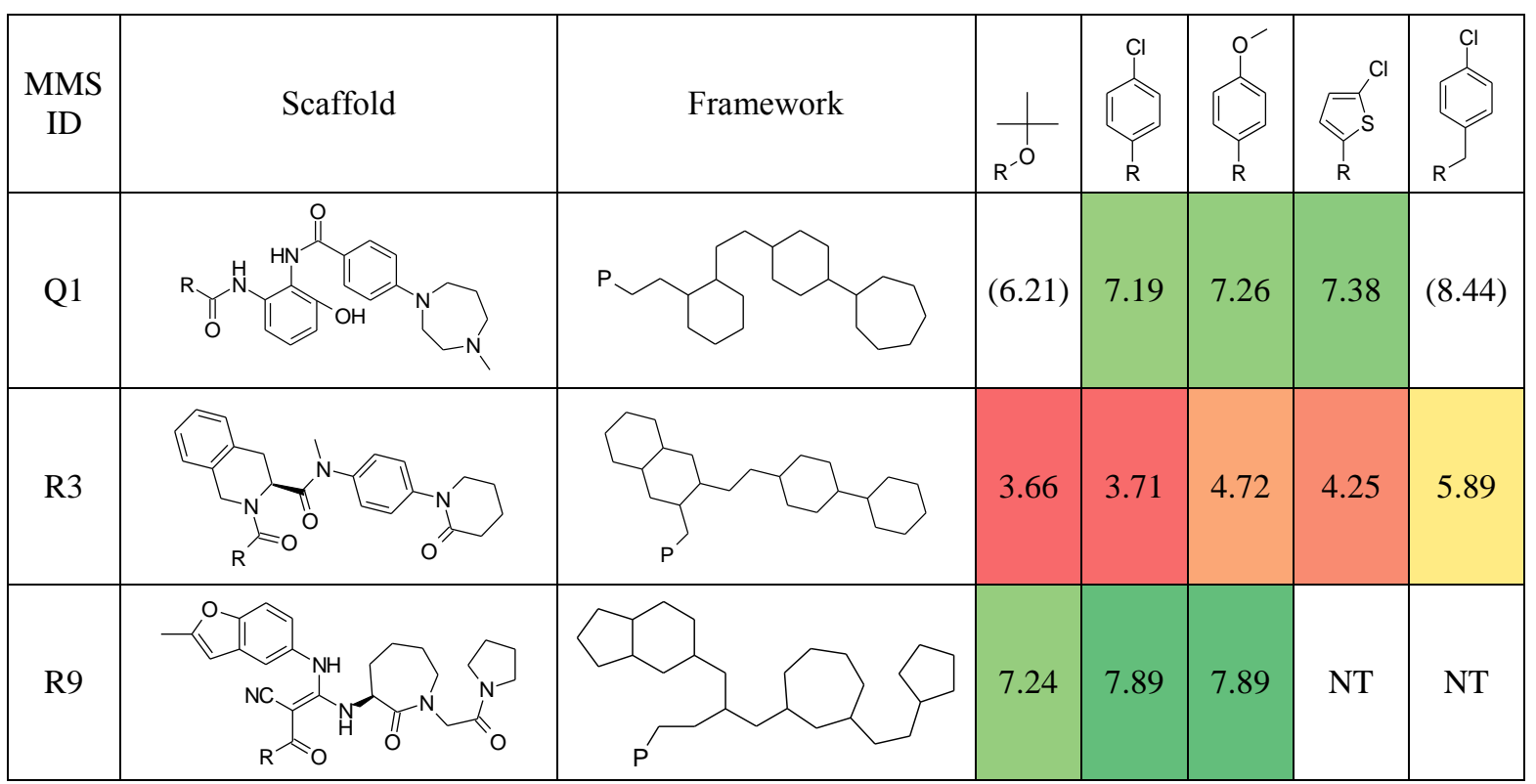

Table 5.j. Representative Fragments with fXa Inhibitory Activities Derived from a Tetrahydroisoquinoline Series

See the corresponding footnotes of Table 5.e.

\begin{tabular}{|c|c|c|c|c|c|c|c|}
\hline $\begin{array}{c}\text { MMS } \\
\text { ID }\end{array}$ & Scaffold & Framework & & cl & $0^{\prime}$ & II & $\mathrm{CF}_{3}$ \\
\hline Q1 & & & 6.46 & 7.19 & 7.26 & $(7.50)$ & (7.68) \\
\hline $\mathrm{R} 2$ & & & 6.43 & 6.53 & 6.64 & 6.31 & NT \\
\hline R10 & & & NT & 5.89 & 4.47 & 3.61 & 3.47 \\
\hline
\end{tabular}


Table 5.k. Representative Fragments with fXa Inhibitory Activities Derived from a Piperidinylsulfonyl Series

See the corresponding footnotes of Table 5.e.

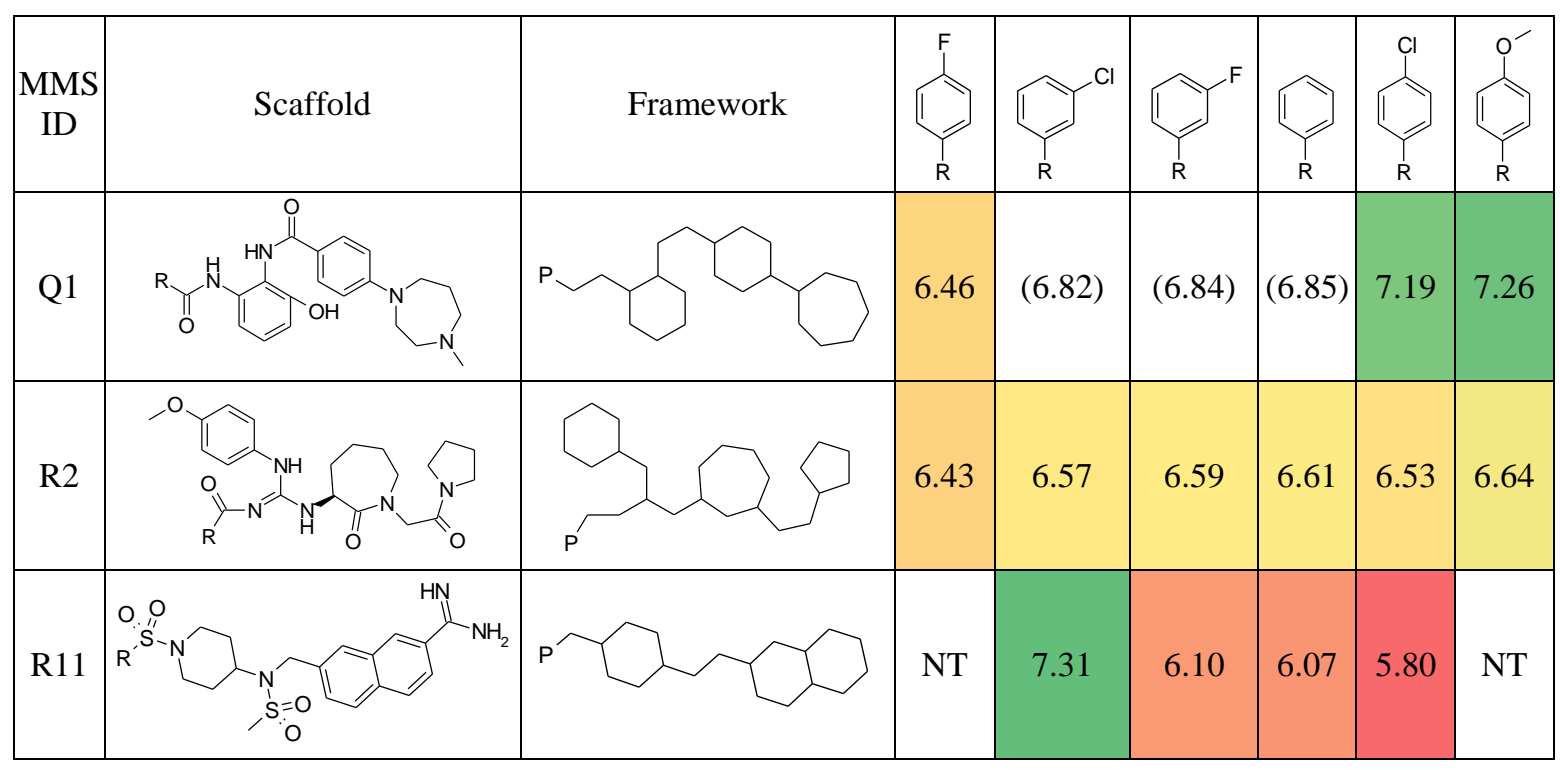

\subsection{Comparison of the Prediction Accuracy of the Fragment Recommendation Workflow with that of a Bayesian Model}

Table 6 summarizes the highest recommendation, according to our workflow, with estimated and measured fXa inhibitory activities. The top recommended fragment was the 3-amidinophenyl group mined from a set of MMS Q1, R3, and R7 (estimated $\mathrm{pIC}_{50}=9.31$, Table 5.g). In contrast, our methodology suggests that a related 4 -amidinophenyl analogue will be less active $\left(\mathrm{pIC}_{50}=\right.$ 6.87) than darexaban.

The predicted order of inhibitory activities of the two amidine derivatives recommended by our workflow was identical to the measured order. We previously reported that a darexaban analogue with a 3-amidinophenyl moiety as an S1 binder exhibited more potent fXa inhibitory activity than darexaban (measured $\mathrm{pIC}_{50}=8.24$ ), although an analogous 4 -amidino isomer was a poor fXa inhibitor with a measured $\mathrm{pIC}_{50}$ value $=4.62$ [40]. However, these data are not registered in ChEMBL version 20, and therefore it is not possible to confirm the applicability of the fragments to the darexaban-chemotype nor to search their fXa inhibitory activities.

The results of a comparative study using a Bayesian classification model were significantly different. The probabilistic Bayesian classification model, which is frequently applied to a binary QSAR model using large SAR databases [30], predicted that the 3- and 4-amidinophenyl derivatives were less potent inhibitors than darexaban (Table 6). These results reveal that our workflow can transfer SAR knowledge and predict activity profiles with high accuracy compared with the standard procedure of the QSAR field. 
Table 6. Top Recommendation for Alternative S1 Binding Elements of fXa Inhibitors Active and inactive mean more and less potent compared with darexaban, respectively.

\begin{tabular}{cccc}
\hline Estimation Method & 9.31 & 7.26 & 6.87 \\
\hline Fragment Recommendation Workflow & Active & Inactive \\
Ensemble Bayesian Classifier & $8.24[40]$ & $6.99[16]$ & $4.62[40]$ \\
Measured Activity & &
\end{tabular}

\section{Conclusion}

Here we report a new drug design workflow that recommends novel compounds with estimated activity profiles. Our protocol comprises sequential steps as follows: (1) preparation of two sets of MMS derived from a query compound collection and reference SAR sources; (2) search for MMS from the reference MMS set, which show profiles similar to a query MMS according to integrated indices derived from 2D-scaffold motifs, including attachment points of fragments, and rank correlation of SAR trends by series of fragments; and (3) analogical estimation using collaborative filtering computation based on standard expertise that activity changes depend on the additive effects of structural modifications. The procedure developed here enables researchers to search direct and indirect relationships to a query MMS queries. We applied our workflow to a retrospective investigation of S1 binding elements of fXa inhibitors, and showed that using SAR and scaffold shape information only, our workflow mined fragments that likely occupy the S1 pocket of fXa. We further show that the accuracy of our method for estimating activity is higher than that of a standard QSAR technique.

\section{References}

[1] Nagahara, T.; Yokoyama, Y.; Inamura, K.; Katakura, S.; Komoriya, S. et al. Dibasic (Amidinoaryl)propanoic Acid Derivatives as Novel Blood Coagulation Factor Xa Inhibitors. J. Med. Chem. 1994, 37, 1200-1207.

[2] Pinto, D. J. P.; Smallheer, J. M.; Cheney, D. L.; Knabb, R. M.; Wexler, R. R. Factor Xa Inhibitors: Next-Generation Antithrombotic Agents. J. Med. Chem. 2010, 53, 6243-6274.

[3] Herron, D. K.; Goodson, T., Jr.; Wiley, M. R.; Weir, L. C.; Kyle, J. A. et al. 1,2-Dibenzamidobenzene Inhibitors of Human Factor Xa. J. Med. Chem. 2000, 43, 859-872.

[4] Yee, Y. K.; Tebbe, A. L.; Linebarger, J. H.; Beight, D. W.; Craft, T. J. et al. $N^{2}$-Aroylanthranilamide Inhibitors of Human Factor Xa. J. Med. Chem. 2000, 43, 873-882.

[5] Masters, J. J.; Franciskovich, J. B.; Tinsley, J. M.; Campbell, C.; Campbell, J. B. et al. Non-Amidine-Containing 1,2-Dibenzamidobenzene Inhibitors of Human Factor Xa with Potent Anticoagulant and Antithrombotic Activity. J. Med. Chem. 2000, 43, 2087-2092.

[6] Gaulton, A.; Bellis, L.; Chambers, J.; Davies, M.; Hersey, A. et al. ChEMBL: a large-scale bioactivity database for drug discovery. Nucleic Acids Res. Database Issue, 2012, 40, D1100-D1107. 
[7] Griffen, E.; Leach, A. G.; Robb, G. R.; Warner, D. J. Matched Molecular Pairs as a Medicinal Chemistry Tool. J. Med. Chem. 2011, 54, 7739-7750.

[8] Gleeson, P.; Bravi, G.; Modi, S.; Lowe, D. ADMET rules of thumb II: A comparison of the effects of common substituents on a range of ADMET parameters. Bioorg. Med. Chem. 2009, 17, 5906-5919.

[9] Warner, D. J.; Griffen, E. J.; St-Gallay, S. A. WizePairZ: A Novel Algorithm to Identify, Encode, and Exploit Matched Molecular Pairs with Unspecified Cores in Medicinal Chemistry. J. Chem. Inf. Model. 2010, 50, 1350-1357.

[10] Keefer, C. E.; Chang, G.; Kauffman, G. W. Extraction of tacit knowledge from large ADME data sets via pairwise analysis. Bioorg. Med. Chem. 2011, 19, 3739-3749.

[11] Ishihara, T.; Mori, K.; Moritomo, A.; Munakata, R.; Matsuda, T. et al. Fragment Map: A Novel System to Search and Visualize Matched Molecular Pairs toward Efficient Multiple ADME/TOX Optimization. Genome Informatics, In press.

[12] Wawer, M.; Bajorath, J. Local Structural Changes, Global Data Views: Graphical Substructure-Activity Relationship Trailing. J. Med. Chem. 2011, 54, 2944-2951.

[13] O’Boyle, N. M.; Bostrom, J.; Sayle, R. A.; Gill, A. Using Matched Molecular Series as a Predictive Tool To Optimize Biological Activity. J. Med. Chem. 2014, 57, 2704-2713.

[14] Gupta-Ostermann, D.; Shanmugasundaram, V.; Bajorath, J. Neighborhood-Based Prediction of Novel Active Compounds from SAR Matrices. J. Chem. Inf. Model. 2014, 54, 801-809.

[15] Ricci, F.; Rokach, L.; Shapira, B. Introduction to Recommender Systems Handbook. In Recommender Systems Handbook, Springer: New York, 2010; pp 1-35.

[16] Hirayama, F.; Koshio, H.; Ishihara, T.; Hachiya, S.; Sugasawa, K. et al. Discovery of N-[2-Hydroxy-6-(4-methoxybenzamido)-phenyl]-4-(4-methyl-1,4-diazepan-1-yl)benzamide (Darexaban, YM150) as a Potent and Orally Available Factor Xa Inhibitor. J. Med. Chem. 2011, 54, 8051-8065.

[17] Dassault Systemes BIOVIA, Pipeline Pilot, Version 9.5, San Diego: Dassault Systemes, 2016.

[18] R Core Team (2016). R: A language and environment for statistical computing. R Foundation for Statistical Computing, Vienna, Austria. https://www.R-project.org/.

[19] Hirayama, F.; Koshio, H.; Katayama, N.; Kurihara, H.; Taniuchi, Y. et al. The Discovery of YM-60828: A Potent, Selective and Orally-Bioavailable Factor Xa Inhibitor. Bioorg. Med. Chem. 2002, 10, 1509-1523.

[20] Hirayama, F.; Koshio, H.; Ishihara, T.; Watanuki, S.; Hachiya, S. et al. Design, synthesis and biological activity of YM-60828 derivatives: potent and orally-Bioavailable factor Xa inhibitors based on naphthoanilide and naphthalensulfonanilide templates. Bioorg. Med. Chem. 2002, 10, 2597-2610.

[21] Koshio, H.; Hirayama, F.; Ishihara, T.; Kaizawa, H.; Shigenaga, T. et al. Orally active factor Xa inhibitor: synthesis and biological activity of masked amidines as prodrugs of novel 1,4-diazepane derivatives. Bioorg. Med. Chem. 2004, 12, 5415-5426.

[22] RDkit: Open-source Cheminformatics. http://www.rdkit.org.

[23] Berthold, M. R.; Cebron, N.; Dill F.; Gabriel, T. R.; Kotter, T. et al. KNIME: The Konstanz Information Miner. In Data Analysis, Machine Learning and Applications, Springer: Berlin, 2007; pp 319-326.

[24] Csardi, G; Nepusz, T. The igraph software package for complex network research, InterJournal Complex Systems 2006, 1695.

[25] Free, S. M. Jr.; Wilson, J. W. A Mathematical Contribution to Structure-Activity Studies. J. Med. Chem. 1964, 7, 395-399.

[26] Kubinyi, H. Free Wilson Analysis. Theory, Applications and its Relationship to Hansch Analysis. Quant. Struct.-Act. Relat. 1988, 7, 121-133. 
[27] Resnick, P.; Iacovou, N.; Suchak, M.; Bergstrom, P.; Riedl, J. In GroupLens: an open architecture for collaborative filtering of netnews, Proceedings of ACM 1994 Conference on Computer Supported Cooperative Work, Chapel Hill, North Carolina, Octber 22-26, 1994.

[28] Lemire, D.; Maclachlan, A. In Slope One Predictors for Online Rating-Based Collaborative Filtering, Proceedings of SIAM Data Mining (SDM'05), Newport Beach, California, April 21-23, 2005.

[29] Kamishima, T. Algorithms for Recommender Systems, Zinkou Tinou Gakkaishi 2008, 23, 89-103.

[30] Glick, N. M.; Davies, J. W.; Jenkins, J. L. Prediction of Biological Targets for Compounds Using Multiple-Category Bayesian Models Trained on Chemogenomics Databases. J. Chem. Inf. Model. 2006, 46, 1124-1133.

[31] Lewell, X. Q.; Judd, D. B.; Watson, S. P.; Hann, M. M. RECAP-Retrosynthetic Combinatorial Analysis Procedure: A Powerful New Technique for Identifying Privileged Molecular Fragments with Useful Applications in Combinatorial Chemistry. J. Chem. Inf. Comput. Sci. 1998, 38, 511-522.

[32] Mochizuki, A.; Nagata, T.; Kanno, H.; Takano, D.; Kishida, M. et al. Orally active zwitterionic factor Xa inhibitors with long duration of action. Bioorg. Med. Chem. Lett. 2011, $21,7337-7343$.

[33] O'Connor, S. P.; Atwal, K.; Li, C.; Liu, E. C.-K.; Seiler, S. M. et al. Synthesis and evaluation of acylguanidine FXa inhibitors. Bioorg. Med. Chem. Lett. 2008, 18, 4696-4699.

[34] Al-Horani, R. A.; Mehta, A. Y.; Desai, U. R. Potent direct inhibitors of factor Xa based on the tetrahydroisoquinoline scaffold. Eur. J. Med. Chem. 2012, 54, 771-783.

[35] Roehrig, S.; Straub, A.; Pohlmann, J.; Lampe, T.; Pernerstorfer, J. et al. Discovery of the Novel Antithrombotic Agent 5-Chloro-N-(\{(5S)-2-oxo-3-[4-(3-oxomorpholin-4-yl)phenyl]1,3-oxazolidin-5-yl \}methyl)thiophene-2-carboxamide (BAY 59-7939): An Oral, Direct Factor Xa Inhibitor. J. Med. Chem. 2005, 48, 5900-5908.

[36] Mochizuki, A.; Nagata, T.; Kanno, H.; Suzuki, M.; Ohta, T. 2-Aminomethylphenylamine as a novel scaffold for factor Xa inhibitor. Bioorg. Med. Chem. 2011, 19, 1623-1642.

[37] Dudley, D. A.; Bunker, A. M.; Chi, L.; Cody, W. L.; Holland, D. R. et al. Rational Design, Synthesis, and Biological Activity of Benzoxazinones as Novel Factor Xa Inhibitors. J. Med. Chem. 2000, 43, 4063-4070.

[38] Shi, Y.; Zhang, J.; Stein, P. D.; Shi, M.; O’Connor, S. P. et al. Ketene aminal-based lactam derivatives as a novel class of orally active FXa inhibitors. Bioorg. Med. Chem. Lett. 2005, 15, 5453-5458.

[39] Ishihara, T.; Seki, N.; Hirayama, F.; Orita, M.; Koshio, H. et al. Prodrug-based design, synthesis, and biological evaluation of N-benzenesulfonylpiperidine derivatives as novel, orally active factor Xa inhibitors. Bioorg. Med. Chem. 2007, 15, 4175-4192.

[40] Koshio, H.; Hirayama, F.; Ishihara, T.; Shiraki, R.; Shigenaga, T. et al. Synthesis and biological activity of novel 1,2-disubstituted benzene derivatives as factor Xa inhibitors. Bioorg. Med. Chem. 2005, 13, 1305-2323. 